\title{
Equivalent Mathematical Representation of Second-Order Damped, Driven Nonlinear Oscillators
}

\author{
Alex Elías-Zúñiga and Oscar Martínez-Romero \\ Centro de Innovación en Diseño y Tecnología, Tecnológico de Monterrey, Campus Monterrey E. Garza Sada 2501 Sur, \\ 64849 Monterrey, NL, Mexico
}

Correspondence should be addressed to Alex Elías-Zúñiga; aelias@itesm.mx

Received 20 May 2013; Accepted 5 September 2013

Academic Editor: Hossein Jafari

Copyright (C) 2013 A. Elías-Zúñiga and O. Martínez-Romero. This is an open access article distributed under the Creative Commons Attribution License, which permits unrestricted use, distribution, and reproduction in any medium, provided the original work is properly cited.

\begin{abstract}
The aim of this paper focuses on applying a nonlinearization method to transform forced, damped nonlinear equations of motion of oscillatory systems into the well-known forced, damped Duffing equation. The accuracy obtained from the derived equivalent equations of motion is evaluated by studying the amplitude-time, the phase portraits, and the continuous wavelet transform diagrams of the cubic-quintic Duffing equation, the generalized pendulum equation, the power-form elastic term oscillator, the Duffing equation with linear and cubic damped terms, and the pendulum equation with a cubic damped term.
\end{abstract}

\section{Introduction}

Here, in this paper, we illustrate how the nonlinearization approach can be used to obtain equivalent equations of motion of forced, damped nonlinear oscillators of the form

$$
\ddot{x}+F(\nu \dot{x})+f(x)+Q\left(\omega_{f} t\right)=0 ; \quad x(0)=A, \quad \dot{x}(0)=0,
$$

where $A$ is the initial oscillation amplitude, $f(x)$ and $F(\nu \dot{x})$ are the system conservative and nonconservative restoring forces, respectively, $v$ is a damping parameter, and $Q\left(\omega_{f} t\right)$ is a periodic external force with driving frequency $\omega_{f}$. In accordance with the nonlinear transformation approach [1], we first write the conservative force terms as a polynomial expression by using the Chebyshev polynomials of the first kind [2-4]:

$$
f(x)=\sum_{n=0}^{N} b_{2 n+1}\left(x_{10}\right) T_{2 n+1}(x),
$$

where

$$
b_{2 n+1}=\frac{2}{\pi} \int_{-1}^{+1} \frac{1}{\sqrt{1-x^{2}}} f(x) T_{2 n+1}(x) d x
$$

and $T_{2 n+1}$ are the Chebyshev polynomials of the first kind. We can see that the usage of (3) could transform (2) into a fifth or higher order polynomial expression. In the case for which a fifth-order Chebyshev polynomial is used, the conservative force in (2) becomes

$$
\begin{aligned}
f(x) & \equiv b_{1}(q) T_{1}(y)+b_{3}(q) T_{3}(y)+b_{5}(q) T_{5}(y) \\
& \approx \alpha(A) x+\beta(A) x^{3}+\gamma(A) x^{5},
\end{aligned}
$$

where $\alpha(A), \beta(A)$ and $\gamma(A)$ will be defined later on. Therefore, the equivalent representation form of (1) is given as

$$
\ddot{x}+F(\nu \dot{x})+\alpha(A) x+\beta(A) x^{3}+\gamma(A) x^{5}+Q\left(\omega_{f} t\right) \approx 0 .
$$

By following the nonlinearization method, we now find the equivalent representation form of (1) as a function of a cubiclike polynomial equation. This procedure leads to

$$
\begin{aligned}
\ddot{x}+ & F(\nu \dot{x})+\alpha(A) x+\beta(A) x^{3}+\gamma(A) x^{5}+Q\left(\omega_{f} t\right) \\
& \simeq \ddot{x}+F\left(\nu_{1} \dot{x}\right)+\delta(A) x+\epsilon(A) x^{3}+Q\left(\omega_{f} t\right),
\end{aligned}
$$


where $\nu_{1}, \delta$, and $\varepsilon$ are determined from

$$
\begin{aligned}
& F_{1}\left(\delta, \epsilon, \nu_{1}, Q\right) \\
& =\int_{0}^{\sigma}\left(F(\nu \dot{x})+\alpha x+\beta x^{3}+\gamma x^{5}+Q\left(\omega_{f} t\right)\right. \\
& \left.\quad-F\left(\nu_{1} \dot{x}\right)-\delta x-\epsilon x^{3}-Q\left(\omega_{f} t\right)\right)^{2} d x \longrightarrow \min \\
& F_{2}\left(\delta, \epsilon, \nu_{1}, Q\right) \\
& =\int_{0}^{v}\left(F(\nu \dot{x})+\alpha x+\beta x^{3}+\gamma x^{5}+Q\left(\omega_{f} t\right)\right. \\
& \left.\quad-F\left(\nu_{1} \dot{x}\right)-\delta x-\epsilon x^{3}-Q\left(\omega_{f} t\right)\right)^{2} d \dot{x} \longrightarrow \min ,
\end{aligned}
$$

in which

$$
\begin{gathered}
\frac{\partial F_{1}\left(\delta, \epsilon, \nu_{1}, Q\right)}{\partial \delta}=0, \quad \frac{\partial F_{1}\left(\delta, \epsilon, \nu_{1}, Q\right)}{\partial \epsilon}=0, \\
\frac{\partial F_{2}\left(\delta, \epsilon, \nu_{1}, Q\right)}{\partial \nu_{1}}=0 .
\end{gathered}
$$

Notice that in our proposed procedure we are assuming that the magnitude of the external force and its driving frequency remain constants during the transformation process. Thus, (1) can be written in equivalent form as

$$
\begin{aligned}
& \frac{d^{2} x}{d t^{2}}+F(\nu \dot{x})+f(x)+Q\left(\omega_{f} t\right) \\
& \quad \equiv \frac{d^{2} x}{d t^{2}}+F\left(\nu_{1} \dot{x}\right)+\delta(A) x+\epsilon(A) x^{3}+Q\left(\omega_{f} t\right) \approx 0 .
\end{aligned}
$$

We will next explore the applicability of our proposed approach and derive the equivalent representation form of some forced, damped nonlinear systems.

\section{The Forced, Damped Cubic-Quintic Oscillator}

The equation of motion that describes the dynamical response of the forced, damped cubic-quintic oscillator is given as

$$
\begin{gathered}
\ddot{y}+2 v \dot{y}+A y+B y^{3}+G y^{5}=Q_{0} \cos \omega_{f} t, \\
\text { with } y(0)=y_{10}, \quad \dot{y}(0)=0,
\end{gathered}
$$

where $y$ denotes the displacement of the system, $v$ is the damping coefficient, $A, B$, and $G$ are system constant parameters, $Q_{0}$ is the magnitude of the external force, and $\omega_{f}$ is the driving frequency $[6,7]$. We next use $x=A / y$ and write (11) as

$$
\begin{gathered}
\ddot{x}+2 \nu \dot{x}+\alpha x+\beta x^{3}+\gamma x^{5}=Q \cos \omega_{f} t, \\
\text { with } x(0)=1, \quad \dot{x}(0)=0,
\end{gathered}
$$

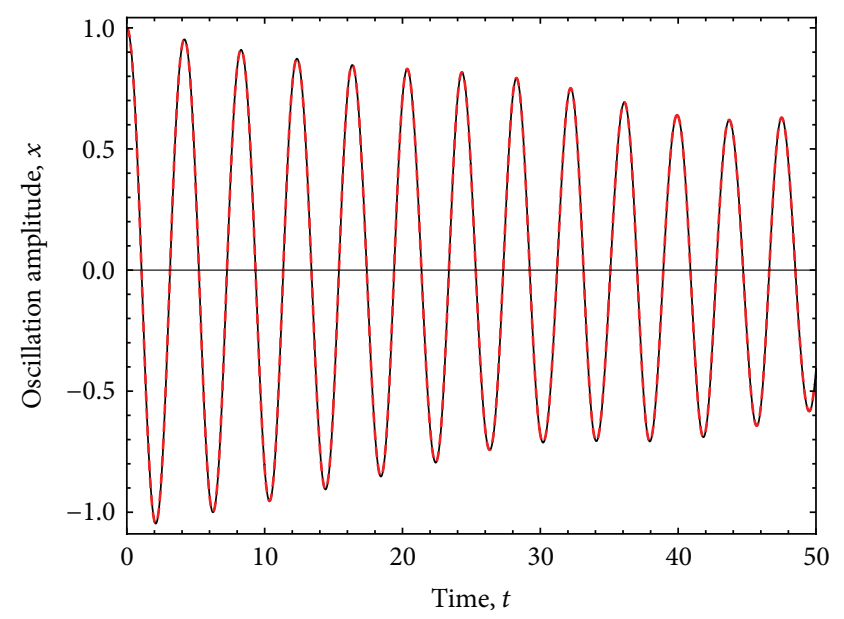

Figure 1: Amplitude-time response curves obtained from the numerical integration solution of (12) and (13) for the system parameter values of $v=0.01, A=3, B=-4, G=1, Q_{0}=0.1$, and $\omega_{f}=3$ with $y(0)=1 / 2$ and $\dot{y}(0)=0$. Here, the black solid line represents the numerical integration solution of (12), while the red dashed line represents the prediction obtained by using the derived equivalent equation of motion (13) with $\delta=2.8713, \epsilon=-0.7178$, $\nu_{1}=0.0101, \sigma=2.1191$, and $v=-350.4$.

where $\alpha=A, \beta=B y_{10}^{2}, \gamma=G y_{10}^{4}$, and $Q=Q_{0} / y_{10}$. By following our proposed nonlinear method and by using (4) and (7)-(9), we obtain the equivalent representation form of (12) as

$$
\ddot{x}+2 v_{1} \dot{x}+\delta x+\epsilon x^{3}=Q \cos \omega_{f} t,
$$

where $\delta, \epsilon$, and $\nu_{1}$ can be determined from the following equations:

$$
\begin{array}{lll}
\delta=\alpha-\frac{25 \gamma \sigma^{4}}{21}, & \epsilon=\beta+\frac{50 \gamma \sigma^{2}}{27}, & \nu_{1}=\nu+\frac{32 \gamma \sigma^{5}}{189 v} \\
\delta=\alpha-\frac{5 \gamma \sigma^{4}}{49}, & \epsilon=\beta+\frac{190 \gamma \sigma^{2}}{189}, & \nu_{1}=\nu-\frac{32 \gamma \sigma^{5}}{1323 v} .
\end{array}
$$

Here, $\sigma$ and $v$ are fitting parameters that satisfy (7)-(8). To examine the accuracy of (13), we next compare its solution with the one obtained from (12) by using the fourth-order Runge-Kutta numerical integration method. Let us consider the parameter values of $y_{10}=1 / 2, v=0.01, A=3, B=-4$, $G=1, Q_{0}=0.1$, and $\omega_{f}=3$. In this case, the parameter values assigned to $A, B$, and $G$ provide a triple-well potential to the cubic-quintic Duffing oscillator that can have up to four resonance frequencies [8]. Figure 1 illustrates the comparison between the amplitude-time response curves of (12) and (13) obtained from their corresponding numerical integration solutions. As one can see form Figure 1, both solutions are almost the same. In fact, the computed root-mean-square error (RMSE) value does not exceed 0.0741 on $0 \leq t \leq 50$ with $\delta=2.8713, \epsilon=-0.7178, \nu_{1}=0.0101, \sigma=2.1191$, and $v=-350.4$. The accuracy of the numerical simulations is 


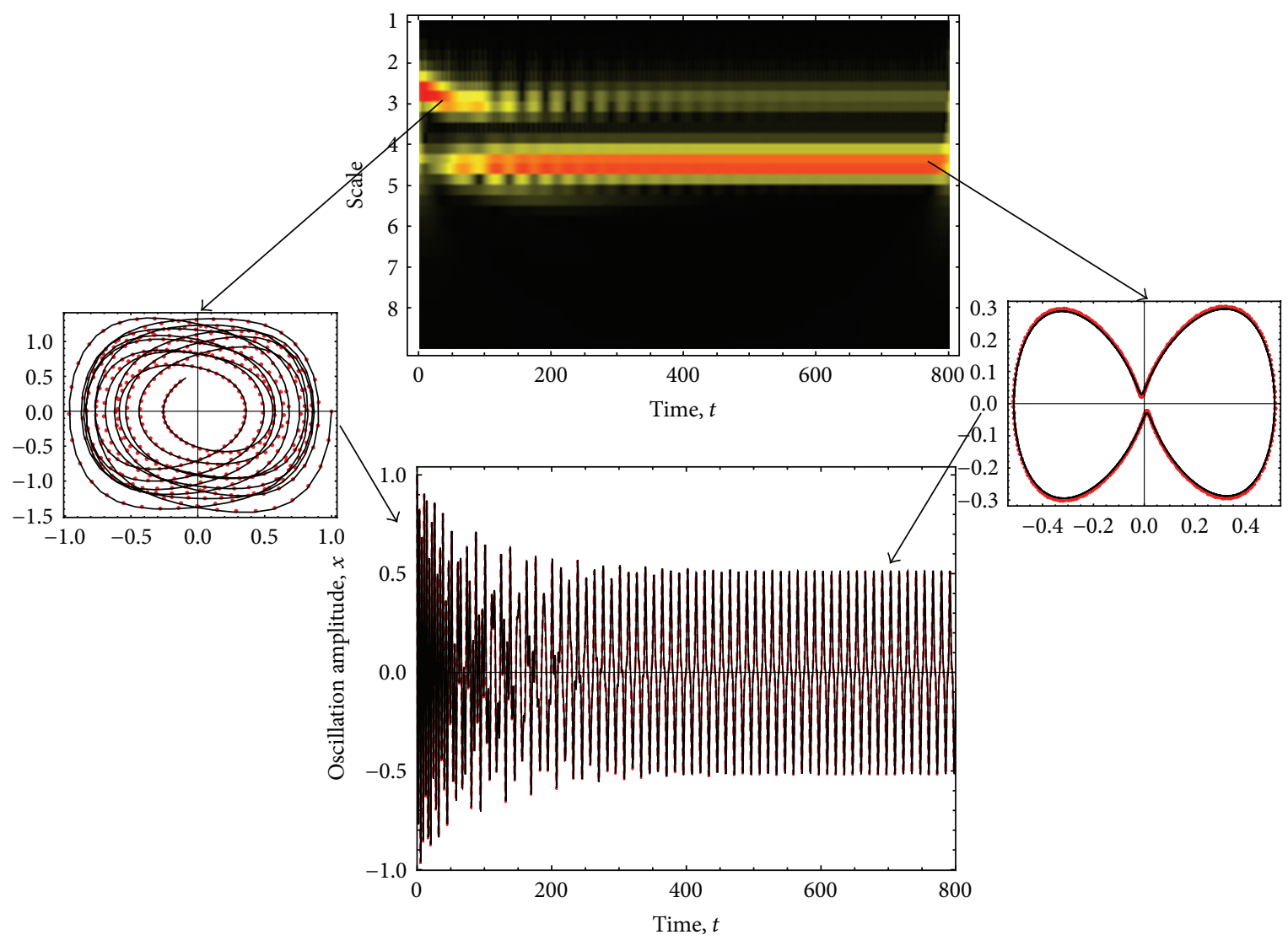

Figure 2: Amplitude-time response curves, phase diagrams, and Morlet CWT plots of (12) and (13), for the system parameter values of $v=0.01, A=1, B=3.5, G=0.5, Q_{0}=0.5, \omega_{f}=0.5$ with $y(0)=1$ and $\dot{y}(0)=0$. Here, the black solid line represents the numerical integration solution of (12) while the red dotted and the red dashed lines represent the prediction obtained by using the derived equivalent equation of motion (13) with $\delta=0.9838, \epsilon=3.7827$, and $\nu_{1}=0.0099$.

surprisingly good if we consider that the potential of a cubic Duffing oscillator cannot have triple-well form.

As a second example, let us consider the parameter values of $y_{10}=1, v=0.01, A=1, B=3.5, G=0.5, Q_{0}=0.5$, and $\omega_{f}=0.5$. Figure 2 shows the amplitude-time response curves and the corresponding phase portraits, as well as the Morlet continuos wavelet transforms (CWT) obtained from the numerical integration solutions of (12) and (13). Here, the values of $\sigma=0.75$ and $v=100$ were computed from (15) which provides good agreement between (12) and (13). Notice that the numerical integration solutions of (12) and (13) are almost the same. In this particular problem, the Morlet CWT was used to extract system dynamics effects such as the one shown at the system transient motion in which the transient frequency has strong influence on the system dynamic behavior. In fact on the time interval $0 \leq t \leq$ 100 , the transient frequency dominates the system motion. When $t>100$, the system oscillates at the driving frequency $\omega_{f}$. Besides, we have computed the RMSE value between both numerical solutions and found that it has the value of 0.0301 . Here, $\delta=0.9838, \epsilon=3.7827$, and $\nu_{1}=0.0099$. Of course, we can consider other parameter values, as those shown in Table 1, to describe the dynamic response of (12) by using (13). Therefore, we can conclude that our nonlinear method leads to the derivation of an equivalent equation of motion that follows well the qualitative and quantitative numerical response of the original equation (12).

We next determine the equivalent representation form of the forced, damped general pendulum equation.

\section{The Forced, Damped General Pendulum Equation}

We now proceed to derive the equivalent representation form of the forced, damped pendulum equation

$$
\begin{gathered}
\frac{d^{2} y}{d t^{2}}+2 v \dot{y}-b y+a \sin y=Q_{0} \cos \left(\omega_{f} t\right), \\
y(0)=A, \quad \dot{y}(0)=0,
\end{gathered}
$$

where $a$ and $b$ represent system constant parameter values, $v$ is the damping coefficient, $\omega_{f}$ is the driving frequency, and $Q_{0}$ is the external force magnitude [9]. If we introduce the transformation $x=y / A$, then (16) can be rewritten as

$$
\begin{aligned}
\frac{d^{2} x}{d t^{2}}+2 v \dot{x}-b x+\frac{a}{A} \sin (x A) & =Q \cos \left(\omega_{f} t\right), \\
x(0)=1, \quad \dot{x}(0) & =0,
\end{aligned}
$$


TABLE 1: Estimated RMSE values computed on the time interval of $0 \leq t \leq 50$ with $\omega_{f}=1$ and $\nu=0.01$.

\begin{tabular}{lccc}
\hline Oscillator & $x_{0}=1 / 2$ & & $x_{0}=1$ \\
& $Q=0.1$ & $Q=1$ & $Q=0.1$ \\
\hline Cubic-quintic duffing oscillator with parameter values of & RMSE & RMSE & RMSE \\
$A=1, B=0.1, G=0.1, \sigma=2.1, v=30$ & 0.0600 & 2.2148 & 0.0967 \\
$A=10, B=10, G=10, \sigma=0.915, v=-350.4$ & 0.0121 & 0.0270 & 0.2948 \\
\hline Pendulum oscillator with parameter values of & & & \\
$a=0.1, b=-5, \sigma=0.8, v=-50$ & 0 & 0 & 0.1147 \\
$a=5, b=-1, \sigma=0.8, v=-50$ & 0 & 0 & 0.00001 \\
\hline
\end{tabular}

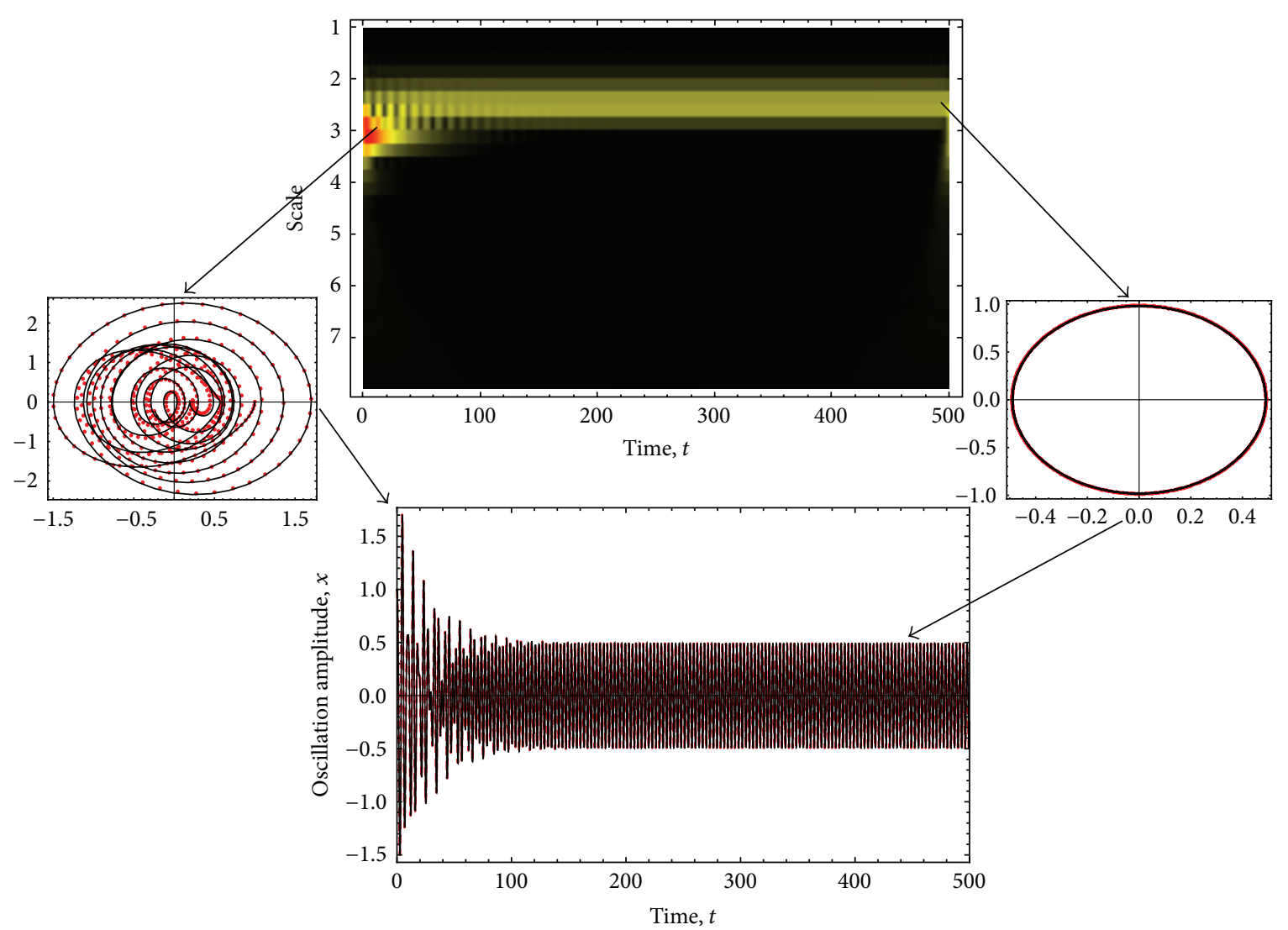

FIgure 3: Amplitude-time response curves, phase diagrams, and Morlet CWR plots of (17) and (18) for the system parameter values of $a=1$, $b=-1, v=0.035, Q=1, \omega_{f}=2$ with $x(0)=57.3^{\circ}$ and $\dot{x}(0)=0$. Here, the black solid line represents the numerical integration solution of (17), while the red dotted and red dashed lines represent the predictions obtained from the derived equivalent equation of motion (18). Here, $\alpha=1.999, \beta=-0.1664, \gamma=0.0079, \delta=1.999, \epsilon=-0.1613$, and $\nu_{1}=0.035$.

with $Q=Q_{0} / A$. By applying our proposed transformation method to (17), we obtain the following expression:

$$
\frac{d^{2} x}{d t^{2}}+2 \nu_{1} \dot{x}+\delta x+\epsilon x^{3}=Q \cos \left(\omega_{f} t\right)
$$

where

$$
\begin{gathered}
\alpha=\frac{6 a}{A^{4}}\left(A\left(A^{2}-80\right) J_{1}(A)-16\left(A^{2}-20\right) J_{2}(A)\right)-b, \\
\beta=\frac{32 a}{A^{4}}\left(-A\left(A^{2}-60\right) J_{1}(A)+2\left(7 A^{2}-120\right) J_{2}(A)\right), \\
\gamma=\frac{32 a}{A^{4}}\left(A\left(A^{2}-48\right) J_{1}(A)-12\left(A^{2}-16\right) J_{2}(A)\right),
\end{gathered}
$$

and $\delta, \epsilon$, and $\nu_{1}$ are given by (14) and (15). Here, $J_{1}(A)$ and $J_{2}(A)$ are the first and second order Bessel functions of the first kind. To illustrate the degree of accuracy attained by our derived solution (18), let us consider the system parameter values of $a=1, b=-1, v=0.035, Q=1$, and $\omega_{f}=2$ with $x(0)=1$ or $57.3^{\circ}$ and $\dot{x}(0)=0$. One can notice from Figure 3 that the numerical integration solutions of (17) and (18) are almost the same. In this case, $\alpha=1.999, \beta=-0.1664, \gamma=$ $0.0079, \delta=1.999, \epsilon=-0.1613$, and $\nu_{1}=0.035$, and the values of $\sigma, v$, and $\nu_{1}$ were fitted by using (15), since these expressions provide the best predictions with a RMSE value of 0.0087 . The same degree of accuracy was found by considering different system parameter values, as those illustrated in Table 1 in which the RMSE values are close to zero. 
To further assess the applicability of our nonlinear cubication approach, we next derive the equivalent representation form of a forced, damped oscillator with a power-form elastic term.

\section{A Generalized Forced, Damped Power-Form Elastic Term Oscillator}

The equation of motion of this oscillator is given as

$$
\begin{aligned}
& \frac{d^{2} y}{d t^{2}}+2 v \frac{d y}{d t}+\omega_{n}^{2} y+h \operatorname{sgn}(y)|y|^{m} \\
& =Q_{0} \cos \left(\omega_{f} t\right), \quad y(0)=A, \quad \dot{y}(0)=0,
\end{aligned}
$$

where $\omega_{n}$ and $h$ are constant parameters and $m$ can take any nonnegative real value, such as odd, even, rational, or irrational, that is, $0 \leq m<\infty$ [10]. As usual, let us use the following coordinate transformation $x=y / A$ and write (20) as,

$$
\begin{aligned}
& \frac{d^{2} x}{d t^{2}}+2 \nu \frac{d x}{d t}+\omega_{n}^{2} x+c_{1} \operatorname{sgn}(x)|x|^{m}=Q \cos \left(\omega_{f} t\right), \\
& c_{1}=h A^{(m-1)} ; \quad Q=\frac{Q_{0}}{A} \quad \text { with } x(0)=1, \quad \dot{x}(0)=0 .
\end{aligned}
$$

We next use Chebyshev polynomial expansion to write the restoring forces $\omega_{n}^{2} x+c_{1} \operatorname{sgn}(x)|x|^{m}$ as a nonic polynomial expression

$$
\omega_{n}^{2} x+c_{1} \operatorname{sgn}(x)|x|^{m} \approx \alpha_{2} x+\beta x^{3}+\gamma x^{5}+\Delta x^{7}+\varepsilon x^{9},
$$

where

$$
\begin{gathered}
\alpha_{2}=\frac{5 c_{1}(m-9)(m-7)(m-5)(m-3) \Gamma[m / 2+1]}{8 \sqrt{\pi} \Gamma[(11+m) / 2]}+\omega_{n}^{2}, \\
\beta=-\frac{10 c_{1}(m-9)(m-7)(m-5)(m-1) \Gamma[m / 2+1]}{\sqrt{\pi} \Gamma[(11+m) / 2]}, \\
\gamma=\frac{42 c_{1}(m-9)(m-7)(m-3)(m-1) \Gamma[m / 2+1]}{\sqrt{\pi} \Gamma[(11+m) / 2]}, \\
\Delta=-\frac{64 c_{1}(m-9)(m-5)(m-3)(m-1) \Gamma[m / 2+1]}{\sqrt{\pi} \Gamma[(11+m) / 2]}, \\
\mathcal{E}=\frac{32 c_{1}(m-7)(m-5)(m-3)(m-1) \Gamma[m / 2+1]}{\sqrt{\pi} \Gamma[(11+m) / 2]} .
\end{gathered}
$$

Notice that in $(23)-(27)$ the terms $\Gamma[m]$ represent the Euler gamma function. It is important to point out that in this particular problem we have used five Chebyshev expansion coefficient terms that provide, for the system restoring force, an equivalent representation form that is based on a ninthorder polynomial expression. This example illustrates the applicability of our procedure in using more than three terms in (4). We next follow our solution procedure and find, by using (7) and (8), that

$$
\begin{aligned}
& \frac{d^{2} x}{d t^{2}}+2 \nu \frac{d x}{d t}+\omega_{n}^{2} x+c_{1} \operatorname{sgn}(x)|x|^{m} \\
& \simeq \frac{d^{2} x}{d t^{2}}+2 v_{1} \frac{d x}{d t}+\delta x+\epsilon x^{3}=Q \cos \left(\omega_{f} t\right)
\end{aligned}
$$

where

$$
\begin{gathered}
\delta=\alpha_{2}-\frac{5\left(715 \gamma \sigma^{4}+1274 \Delta \sigma^{6}+1701 \varepsilon \sigma^{8}\right)}{3003}, \\
\epsilon=\beta+\frac{50 \gamma \sigma^{2}}{27}+\frac{245 \Delta \sigma^{4}}{99}+\frac{420 \varepsilon \sigma^{6}}{143}, \\
\nu_{1}=\nu+\frac{32\left(143 \gamma \sigma^{5}+273 \Delta \sigma^{7}+378 \varepsilon \sigma^{9}\right)}{27027 v}, \\
\delta=\alpha_{2}-\frac{5 \gamma \sigma^{4}}{49}-\frac{10 \Delta \sigma^{6}}{231}+\frac{45 \varepsilon \sigma^{8}}{1001}, \\
\epsilon=\beta+\frac{190 \gamma \sigma^{2}}{189}+\frac{85 \Delta \sigma^{4}}{99}+\frac{100 \varepsilon \sigma^{6}}{143} \\
\nu_{1}=\frac{189189 v v-32\left(143 \gamma \sigma^{5}+273 \Delta \sigma^{7}+378 \varepsilon \sigma^{9}\right)}{189189 v} .
\end{gathered}
$$

To assess the accuracy of our derived equivalent representation form (28) of (21), we shall consider the following data values: $m=8 / 5, A=1, \omega_{n}=0, h=10, Q=10$, and $v=0.1$ with a driving frequency value of $\omega_{f}=3$ [11]. Figure 4 illustrates the amplitude-time response curves obtained by numerically integrating (21) and (28). As we can see from Figure 4, the numerical integration of (28) follows closely the amplitude-time response curve obtained from (21). In this case, the RMSE value of 0.384 is obtained by using equations (30)-(32). Here, the red solid and black dashed lines represent, respectively, the numerical integration solution of (21) and (28). The computed parameter values are $\alpha_{2}=3.0954, \beta=21.2256, \gamma=-36.7078, \Delta=$ $35.2187, \varepsilon=-12.8501, \delta=3.5311, \epsilon=3.6588, v_{1}=$ $0.1155, v=-50$, and $\sigma=-1.22$. Also, Figure 5 provides a comparison of the numerical solutions of (21) and (28) with respect to the approximate general solution of (28) derived by using Jacobi elliptic functions [5]. One can notice from Figure 5 that all solutions are almost the same. Therefore, we can conclude that our derived equivalent representation form (28) describes well the qualitative and quantitative behavior of (21). The amplitude-frequency response curve of (28) can be obtained by using, for instance, the approximate solutions developed in $[5,11]$. As a second case, we now use our equivalent representation form (28) and consider the following parameter values of $m=1 / 3, A=1, \omega_{n}=2, h=$ $0.1, Q=0.1, \nu=0.025$, and $\omega_{f}=2$ in (21) and compute the corresponding amplitude-time response curve. We can see from Figure 6 that the amplitude-time curve obtained from (28) follows well the curve obtained from (21). In this case, the 


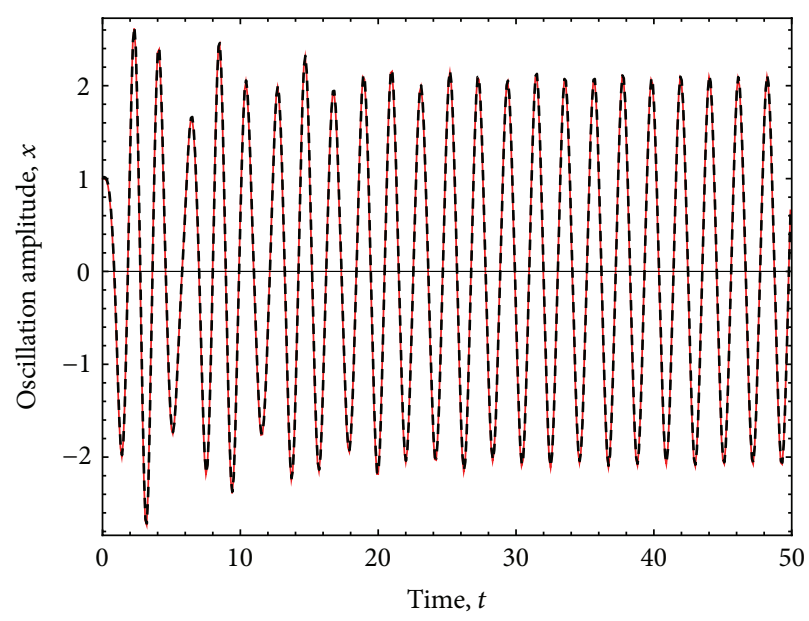

(a)

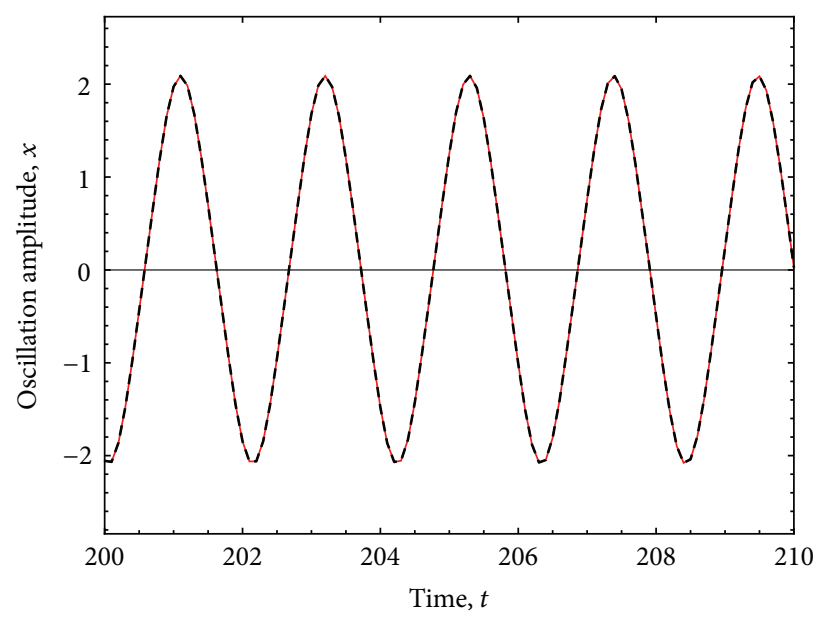

(b)

Figure 4: Amplitude-time response curves of (21) and (28) for the system parameter values of $m=8 / 5, A=1, \omega_{n}=0, h=10, Q=10$, $\nu=0.1$, and $\omega_{f}=3$ with $x(0)=1$ and $\dot{x}(0)=0$. Here, the red solid and black dashed lines represent, respectively, the numerical integration solution of (21) and (28) with $\alpha_{2}=3.0954, \beta=21.2256, \gamma=-36.7078, \Delta=35.2187, \varepsilon=-12.8501, \delta=3.5311, \epsilon=3.6588, v_{1}=0.1155$, $v=-50$, and $\sigma=-1.22$.

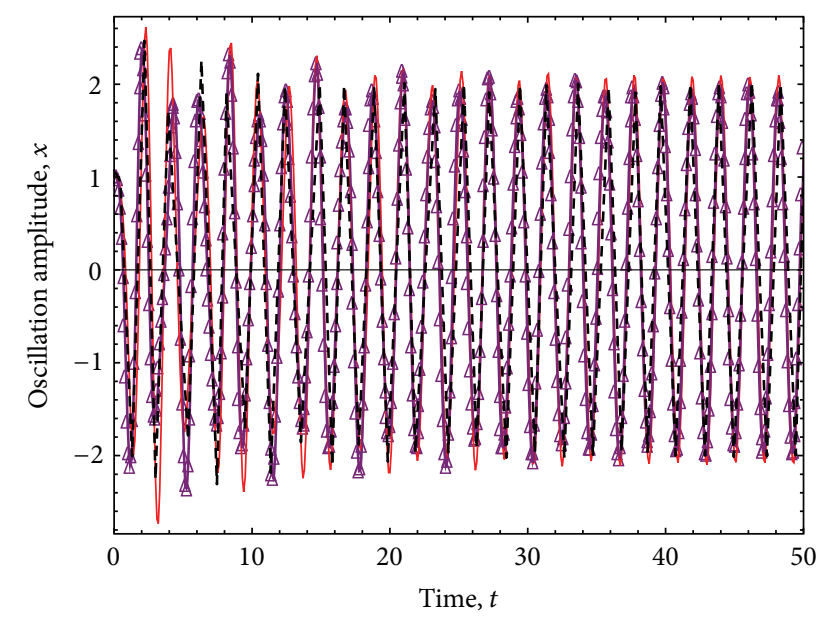

FIgure 5: Amplitude-time response curves of (21) and (28) for the system parameter values of $m=8 / 5, A=1, \omega_{n}=0, h=10, Q=10$, $v=0.1$, and $\omega_{f}=3$ with $x(0)=1$ and $\dot{x}(0)=0$. Here, the red solid and black dashed lines represent, respectively, the numerical integration solution of (21) and (28), while the purple triangles represent the approximate solution of (21) derived in [5] with $\alpha_{2}=$ 3.0954, $\beta=21.2256, \gamma=-36.7078, \Delta=35.2187, \varepsilon=-12.8501$, $\delta=3.5311, \epsilon=3.6588, v_{1}=0.1155, v=-50$, and $\sigma=-1.22$.

RMSE value is about 0.0219 for which the parameter values are $\alpha_{2}=4.3426, \beta=-1.3704, \gamma=3.289, \Delta=-3.508, \varepsilon=$ $1.3493, \delta=4.2695, \epsilon=-0.2956, v_{1}=0.2537, v=-10$, and $\sigma=0.75$. For illustrative purposes, we show in Table 2 some values of the exponent $m$ with their fitting parameter values of $\sigma$ and $v$ that can be used to study the dynamical behavior of some nonlinear oscillator with a rational or irrational power restoring forces.

We next develop the equivalent representation form of the Duffing equation with linear and cubic damped terms.

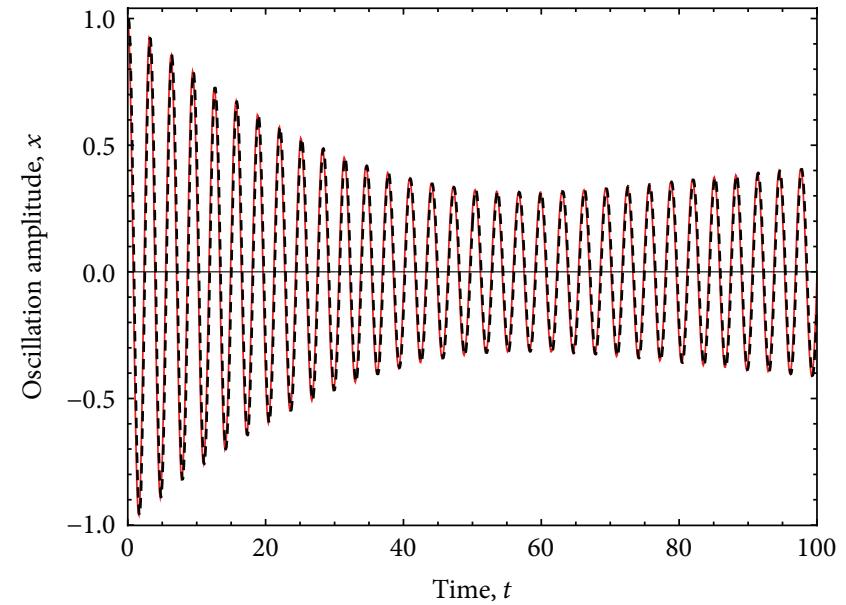

Figure 6: Amplitude-time response curves of (21) and (28) for the system parameter values of $m=1 / 3, A=1, \omega_{n}=2, h=0.1, Q=0.1$, $v=0.025$, and $\omega_{f}=2$ with $x(0)=1$ and $\dot{x}(0)=0$. Here, the red solid and black dashed lines represent, respectively, the numerical integration solution of (21) and (28) with $\alpha_{2}=4.3426, \beta=-1.3704$, $\gamma=3.289, \Delta=-3.508, \varepsilon=1.3493, \delta=4.2695, \epsilon=-0.2956$, $\nu_{1}=0.2537, v=-10$, and $\sigma=0.75$.

\section{The Forced Duffing Equation with Linear and Cubic Damped Terms}

We now explore the applicability of our method to derive the equivalent representation form of the following equation of motion

$$
\begin{gathered}
\frac{d^{2} y}{d t^{2}}+v \dot{y}+A y+B_{0} y^{3}+\kappa_{0} \dot{y}^{3}=Q_{0} \cos \omega_{f} t, \\
y(0)=y_{10}, \quad \dot{y}(0)=\dot{y}_{10},
\end{gathered}
$$


TABLE 2: Estimated values of the fitting parameter $\sigma$ and $v$ at different exponent values of $m$. Here, we assume that $\omega_{n}=2, h=0.1$, $\nu=0.025, Q=0.1$ and consider the following interval values of the initial oscillation amplitude $0.1 \leq A \leq 10$, with driving frequency values on $0.1 \leq \omega_{f} \leq 3$ to best fit the values of $\sigma$ and $v$.

\begin{tabular}{lcc}
\hline $\begin{array}{l}\text { Exponent } \\
\text { value }\end{array}$ & $\begin{array}{c}\text { Fitting Parameter } \\
\text { value }\end{array}$ & $\begin{array}{c}\text { Fitting Parameter } \\
\text { value }\end{array}$ \\
$m$ & $\sigma$ & $v$ \\
\hline $3 / 5$ & -1.1 & 50 \\
$2 / 3$ & -0.95 & 50 \\
$4 / 3$ & -1.177 & -50 \\
$5 / 3$ & -0.95 & 5 \\
$7 / 5$ & -0.95 & 5 \\
$10 / 7$ & 0.9 & 1 \\
\hline
\end{tabular}

which has a linear damped term, $\nu$, and a cubic one, $\kappa_{0}[12,13]$. Let $x=y / y_{10}$; then, (33) can be written as

$$
\begin{gathered}
\frac{d^{2} x}{d t^{2}}+v \dot{x}+A x+B x^{3}+\kappa \dot{x}^{3}=Q \cos \omega_{f} t, \\
x(0)=1, \quad \dot{x}(0)=\dot{x}_{10},
\end{gathered}
$$

where $B=B_{0} y_{10}^{2}, \kappa=\kappa_{0} y_{10}^{2}$, and $Q=Q_{0} / y_{10}$.

Since (34) has a damped nonlinear term of the cubic-type, we need to modify our nonlinear method to take into account its effects on the solution response of (34). Therefore, we now assume that (7) and (8) can be re-written as

$$
\begin{gathered}
F_{1}\left(\delta, \epsilon, v_{1}\right) \\
=\int_{0}^{\sigma}\left(v \dot{x}+A x+B x^{3}+\kappa \dot{x}^{3}+Q \cos \left(\omega_{f} t\right)\right. \\
\quad-\left(\kappa\left|v_{1}\right|+\nu\right) \dot{x}-\delta x \\
\left.\quad-\epsilon x^{3}-Q \cos \left(\omega_{f} t\right)\right)^{2} d x \longrightarrow \min \\
F_{2}\left(\delta, \epsilon, v_{1}\right) \\
=\int_{0}^{v}\left(v \dot{x}+A x+B x^{3}+\kappa \dot{x}^{3}+Q \cos \left(\omega_{f} t\right)\right. \\
-\left(\kappa\left|v_{1}\right|+\nu\right) \dot{x}-\delta x-\epsilon x^{3} \\
\left.-Q \cos \left(\omega_{f} t\right)\right)^{2} d \dot{x} \longrightarrow \min ,
\end{gathered}
$$

which yield the equivalent representation form of (34) as

$$
\frac{d^{2} x}{d t^{2}}+\left(\kappa\left|\nu_{1}\right|+\nu\right) \dot{x}+\delta x+\epsilon x^{3}=Q \cos \left(\omega_{f} t\right)
$$

where

$$
\delta=\frac{18 \kappa v^{3}+A \sigma}{\sigma}, \quad \epsilon=\frac{B \sigma^{3}-14 \kappa v^{3}}{\sigma^{3}}, \quad \nu_{1}=\frac{27 v^{2}}{5}
$$

Before we evaluate the accuracy achieved by our derived expression (37), we first recall that Trueba and coworkers in [13], by using Melnikov analysis, found an equivalent equation of motion for (34) given as

$$
\frac{d^{2} x}{d t^{2}}+\mu \dot{x}+A x+B x^{3}=Q \cos \omega_{f} t
$$

where $\mu$ is defined as

$$
\mu=\nu+\frac{12}{35} \kappa
$$

In what follows, we will use (37) and (39) to compare their numerical predictions with those provided by (34). First, let us consider the parameter values of $v=0.1, A=-1$, $B=1, \kappa=0.01, \omega_{f}=1$, and $Q=0.075$ with $x_{10}=$ 1 , and $\dot{x}_{10}=-1.1463$ and use our derived expressions to compute the values of $\delta, \epsilon, \nu_{1}, \sigma$, and $v$ which are given as $-0.9906,0.9858,0.6060,0.72$, and 0.335 , respectively. Figure 7 shows a comparison of the amplitude-time curves, the phase portrait plots, and the Morlet CWT diagram obtained from the numerical integrations of (34), (37), and (39). Notice from Figure 7, that our equivalent equation of motion (37) closely follows the numerical integration curve of (34). Here, the RMSE value is close to 0.082, while the numerical predictions obtained from (39) show some discrepancies in the amplitude-time curve at the time interval of $30 \leq t \leq 60$. In this solution, the computed RMSE value is 0.199 .

As a second case, we now explore the accuracy of our equivalent representation form (37) by assuming that $\nu=0.2$, $A=2, B=5, \kappa=0.15, \omega_{f}=1 / 2$, and $Q=5$ with $x_{10}=1$ and $\dot{x}_{10}=1$. As we can see from Figure 8 , the numerical integration solutions of (37) and (39) agree well with the solution of (34). Furthermore, the Morlet CWT of (39) shown in Figure 8, exhibits the subharmonic effects that the original system (34) experiences at the frequencies values of $1 / 2 \omega_{f}, 1 / 4 \omega_{f}$, and $1 / 8 \omega_{f}$ which correspond to the Morlet continuous wavelet transform scale values of 3,4 , and 5 , respectively. In this case, the estimated RMSE values are 0.009 for (37) and 0.0166 for (39). The values of $\delta, \epsilon, \nu_{1}, \sigma$, and $v$ are found to be $2.1409,4.7884,0.6060,-0.72$, and 0.335 . Notice that in both examples the values of $v_{1}=0.6060, \sigma=0.72$, and $v=0.335$ remain unchanged. In Figures 7 and 8 , the black and the red dashed lines describe the amplitude-time and phase portrait curves of (34) and (39), respectively.

As a final example, we now derive the equivalent equation of the forced pendulum equation with a cubic damped term

$$
\begin{aligned}
& \frac{d^{2} x}{d t^{2}}+v \dot{x}+\kappa \dot{x}^{3}+a \sin (x A) \\
& =Q \cos \left(\omega_{f} t\right), \quad x(0)=1, \quad \dot{x}(0)=\dot{x}_{10},
\end{aligned}
$$

where $A$ is the initial oscillation amplitude, $a=a_{0} / A, \kappa=$ $\kappa_{0} A^{2}$, with $Q=Q_{0} / A$. We first use Chebyshev polynomial expansion and then (35) and (36) to get that

$$
\frac{d^{2} x}{d t^{2}}+\left(\kappa\left|\nu_{1}\right|+\nu\right) \dot{x}+\delta x+\epsilon x^{3}=Q \cos \left(\omega_{f} t\right)
$$




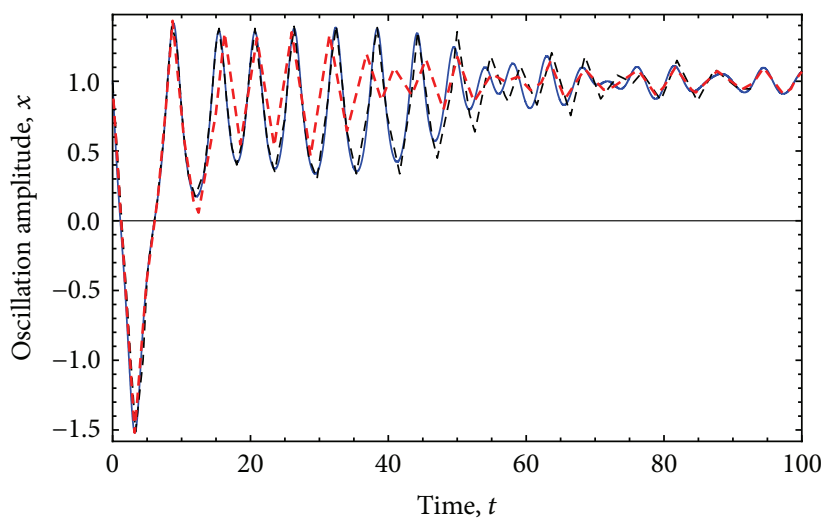

(a)

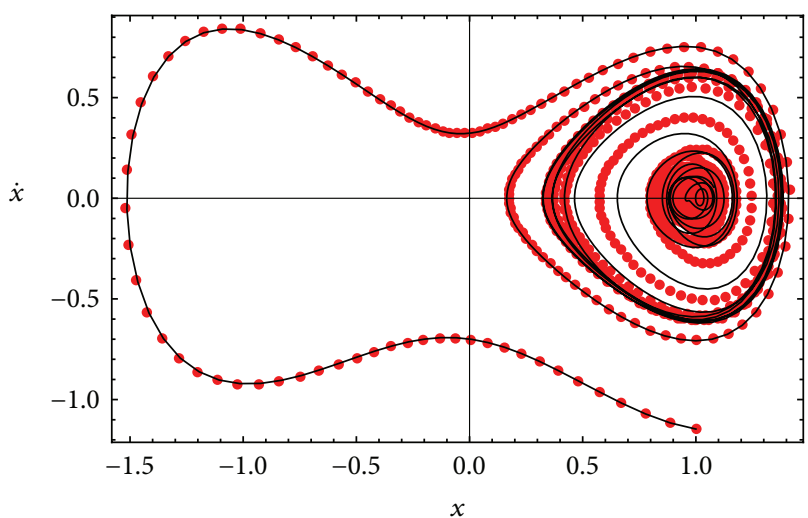

(b)

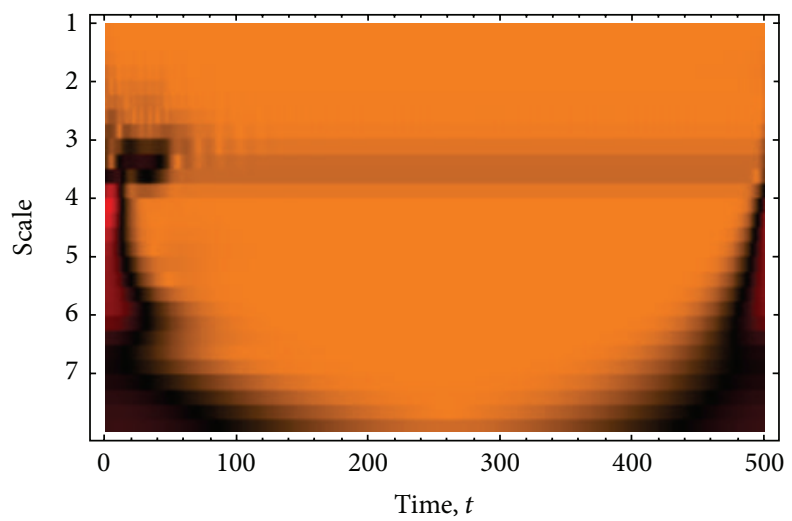

(c)

Figure 7: Amplitude-time, phase plane, and Morlet CWT diagrams for the system parameter values of $\nu=0.1, A=-1, B=1, \kappa=0.01$, $\omega_{f}=1$, and $Q=0.075$ with $x_{10}=1$ and $\dot{x}_{10}=-1.1463$. Here, the dashed black and red lines represent the numerical integration solutions of (34) and (39), while the blue solid line describes the numerical integration solution of (37). Similarly, in (b) the black solid line and the red dots represent the numerical integration solutions computed respectively, from (34) and (37).

where

$$
\begin{gathered}
\alpha=\frac{6 a}{A^{3}}\left(A\left(A^{2}-80\right) J_{1}(A)-16\left(A^{2}-20\right) J_{2}(A)\right)-b, \\
\beta=\frac{32 a}{A^{3}}\left(-A\left(A^{2}-60\right) J_{1}(A)+2\left(7 A^{2}-120\right) J_{2}(A)\right), \\
\gamma=\frac{32 a}{A^{3}}\left(A\left(A^{2}-48\right) J_{1}(A)-12\left(A^{2}-16\right) J_{2}(A)\right),
\end{gathered}
$$

and $\delta, \epsilon$, and $\nu_{1}$ are determined from

$$
\begin{gathered}
\delta=\alpha+\frac{18 \kappa v^{3}}{\sigma}-\frac{185 \gamma \sigma^{4}}{21}, \\
\epsilon=\beta-\frac{14 \kappa v^{3}}{\sigma^{3}}+\frac{70 \gamma \sigma^{2}}{9} \\
\nu_{1}=\frac{567 \kappa v^{3}-320 \gamma \sigma^{5}}{105 \kappa v} .
\end{gathered}
$$

By using Melnikov analysis, Trueba and coworkers [13] developed the equivalent representation form of (41) which is given as

$$
\frac{d^{2} x}{d t^{2}}+\mu \dot{x}+a \sin (x A)=Q \cos \left(\omega_{f} t\right), \quad \mu=\nu+\frac{8}{3} \kappa .
$$

We next consider the parameter values of $A=1, a_{0}=1$, $\nu=0.05, \kappa_{0}=0.1, Q_{0}=0.41, \dot{x}_{10}=1$, and $\omega_{f}=2.5$, and plot the numerical integration solutions of (41), (42), and (45). The corresponding amplitude-time, phase portrait, and Morlet CWT plots are shown in Figure 9 for which the computed parameter values are $\delta=0.9531, \epsilon=-0.1048$, $\nu_{1}=0.615, \alpha=0.999, \beta=-0.1664, \gamma=0.0079, \mu=0.3166$, with $\sigma=0.8$, and $v=-0.2$. We can see from Figure 9 that our solution closely follows the numerical simulations of (41). In this case, the computed RMSE values from (42) and (45) are 0.0893 and 0.2556 , respectively. In Figure 9, the black, the purple, and the red solid lines represent, respectively, the numerical integration solutions of (41), (42), and (45).

This confirms the usefulness of our proposed nonlinear method to obtain equivalent equations of motion of nonlinear oscillators. 


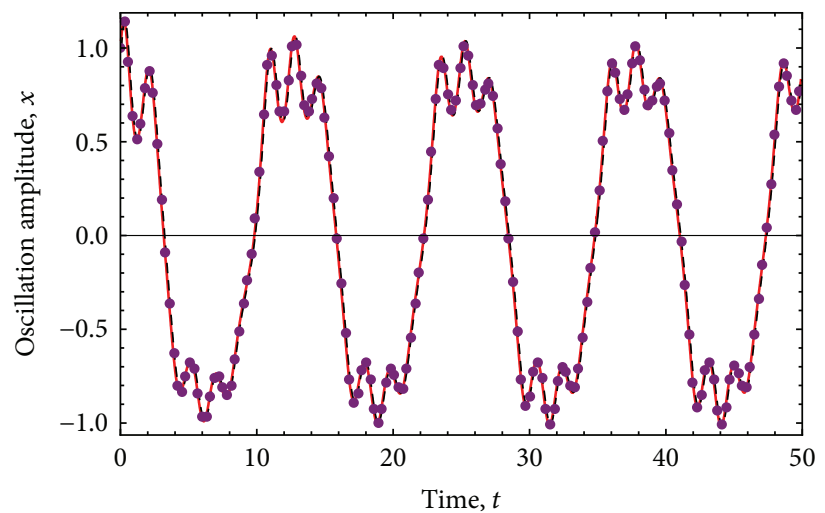

(a)

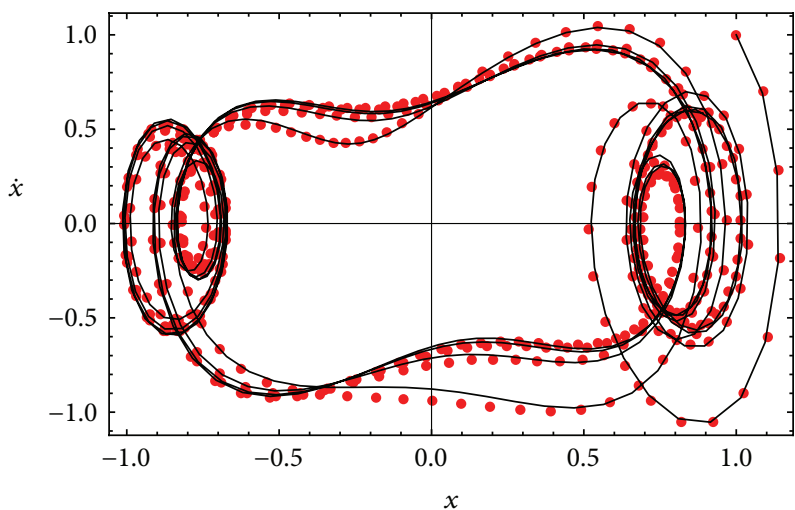

(b)

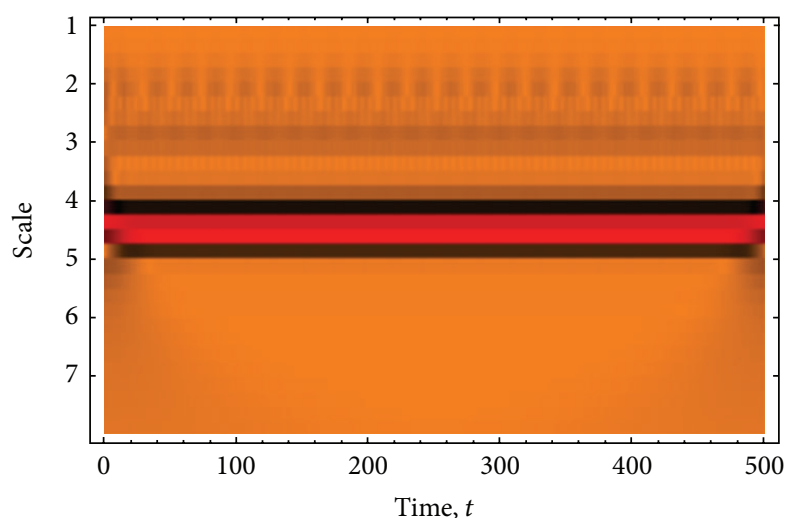

(c)

Figure 8: Amplitude-time, phase plane, and Morlet CWT diagrams for the system parameter values of $\nu=0.2, A=2, B=5, \kappa=0.15$, $\omega_{f}=0.5$, and $Q=5$ with $x_{10}=1$ and $\dot{x}_{10}=1$. Here, the dashed black and red lines represent the numerical integration solutions of ( 34$)$ and (39), while the blue dots describe the numerical integration solution of (37). Similarly, in (b) the black solid line and the red dots represent the numerical integration solutions computed respectively, from (34) and (37).

\section{Conclusions}

In this paper, we have obtained the equivalent representation form of some driven, damped nonlinear oscillators by using a nonlinearization approach. We have found that in all cases, the numerical predictions obtained from the corresponding equivalent representation form of the cubicquintic, the general pendulum, the power-form elastic term, and the cubic damped nonlinear oscillators describe well the qualitative and quantitative behavior of their original equations of motion.

During the solution processes of the forced Duffing equation with a cubic damped term, we have found that our equivalent solution (37) provides numerical estimates that are similar to those obtained from (39) which was derived from Melnikov analysis in [13]. Besides, the numerical predictions of (37) are closer to the numerical integration values of (34) than those obtained from (39). In this oscillator, we have found that the values of $\nu_{1}=0.6060, \sigma=0.72$, and $v=0.335$ could remain unchanged even at larger values of $\nu, A, B, \kappa$, and $Q$. To further evaluate the accuracy of our proposed method, we have developed the solution of a pendulum equation with a cubic damped term and compared its numerical estimated values with those obtained from the original equation of motion and with respect to those of its equivalent representation form derived by Melnikov analysis [13]. We found that our derived equation (42) describes well the numerical estimated values of (41). Moreover, and based on the numerical simulations performed on the last example of this work, it is clear that the derived equivalent equation of motion, when compared to other solutions such as the one derived by using the well-established Melnikov analysis, exhibits good accuracy for a wide range of system parameter values [13]. Of course, one must be careful when using the equivalent transformation forms previously derived, since their degree of accuracy depends not only on the system parameter values but also on the method used to replace the corresponding restoring forces for equivalent ones of the cubic type.

Finally, it is evident that our proposed nonlinearization method can be used to derive equivalent representation forms of other nonlinear oscillators such as the ones examined by the authors in $[14,15]$ and references cited therein, in which rational or irrational restoring forces, as well as damping terms, are used to model the dynamics behavior of common problems that arise in the physical sciences and engineering fields. 


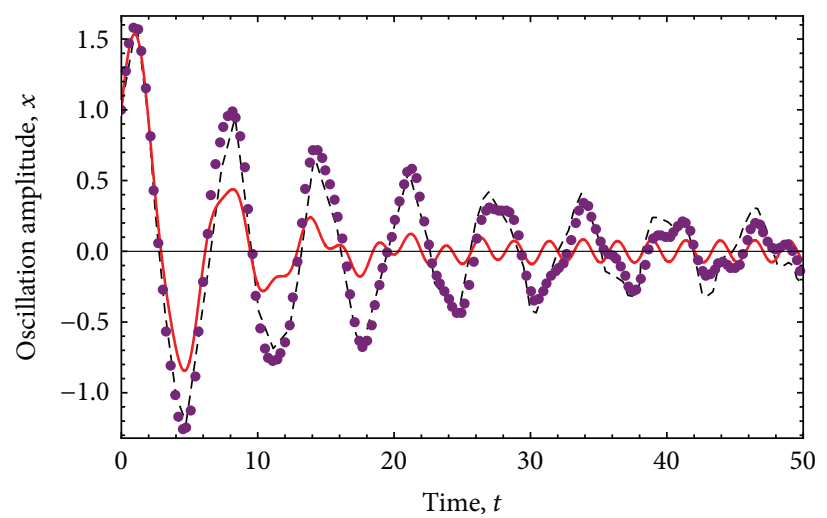

(a)

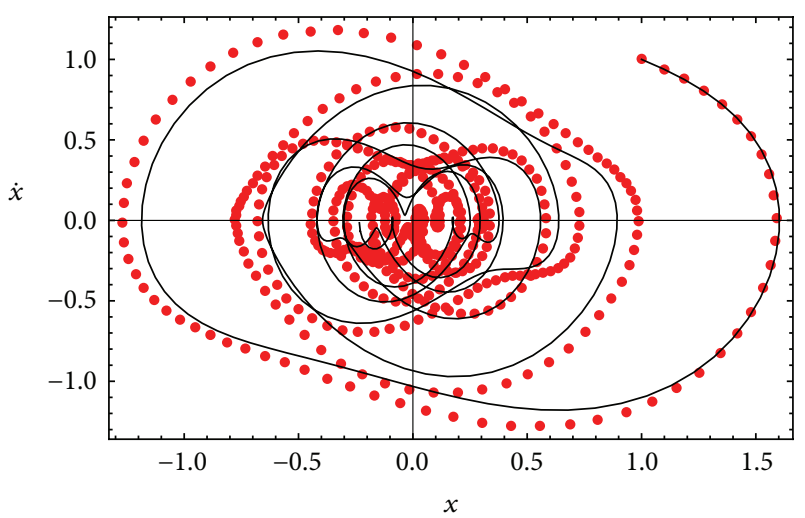

(b)

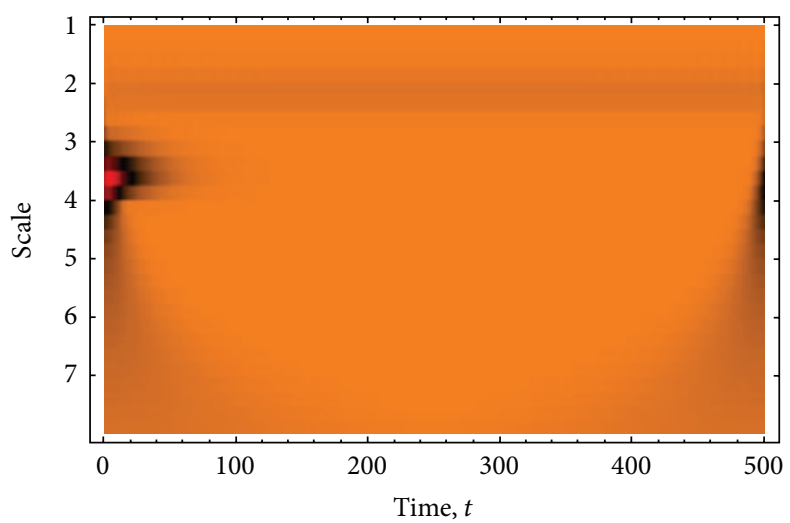

(c)

Figure 9: Amplitude-time, phase plane, and Morlet CWT diagrams for system parameter values of $A=1, a_{0}=1, v=0.05, \kappa_{0}=0.1$, $Q_{0}=0.41, \dot{x}_{10}=1$, and $\omega_{f}=2.5$. Here, the black, the purple, and the red lines represent, respectively, the numerical integration solutions of (41), (42), and (45). Similarly, in (b) the black solid line and the red dots represent the numerical integration solutions computed respectively, from (41) and (42).

\section{Acknowledgments}

This work was funded by the Tecnológico de Monterrey, Campus Monterrey -Campus Monterrey, through the Research Chair in Nanomaterials for Medical Devices and Research Chair in Intelligent Machines. Additional support was provided by the European Union Seventh Framework Programme (FP7-PEOPLE-2009) under the grant agreement IRSES no. 247476 and from Consejo Nacional de Ciencia y Tecnología (Conacyt), México.

\section{References}

[1] A. Elías-Zúñiga, O. Martínez-Romero, and R. K. Córdoba-Díaz, "Approximate solution for the Duffing-harmonic oscillator by the enhanced cubication method," Mathematical Problems in Engineering, Article ID 618750, 12 pages, 2012.

[2] A. Beléndez, M. L. Lvarez, E. Fernndez, and I. Pascual, "Cubication of conservative nonlinear oscillators," European Journal of Physics, vol. 30, no. 5, pp. 973-981, 2009.

[3] A. Beléndez, D. I. Méndez, E. Fernández, S. Marini, and I. Pascual, "An explicit approximate solution to the Duffingharmonic oscillator by a cubication method," Physics Letters,
Section A: General, Atomic and Solid State Physics, vol. 373, no. 32, pp. 2805-2809, 2009.

[4] A. Beléndez, G. Bernabeu, J. Francés, D. I. Méndez, and S. Marini, "An accurate closed-form approximate solution for the quintic Duffing oscillator equation," Mathematical and Computer Modelling, vol. 52, no. 3-4, pp. 637-641, 2010.

[5] A. Elías-Zúñiga, "A general solution of the Duffing equation," Nonlinear Dynamics, vol. 45, no. 3-4, pp. 227-235, 2006.

[6] M. S. Siewe and U. H. Hegazy, "Homoclinic bifurcation and chaos control in MEMS resonators," Applied Mathematical Modelling. Simulation and Computation for Engineering and Environmental Systems, vol. 35, no. 12, pp. 5533-5552, 2011.

[7] M. Taylan, "The effect of nonlinear damping and restoring in ship rolling," Ocean Engineering, vol. 27, no. 9, pp. 921-932, 2000.

[8] S. Jeyakumari, V. Chinnathambi, S. Rajasekar, and M. A. F. Sanjuan, "Analysis of vibrational resonance in a quintic oscillator," Chaos, vol. 19, no. 4, Article ID 043128, 2009.

[9] E. I. Butikov, "Extraordinary oscillations of an ordinary forced pendulum," European Journal of Physics, vol. 29, no. 2, pp. 215233, 2008.

[10] V. N. Pilipchuk, "Analytical study of vibrating systems with strong non-linearities by employing saw-tooth time transformations," Journal of Sound and Vibration, vol. 192, no. 1, pp. 4364, 1996. 
[11] I. Kovacic, "Forced vibrations of oscillators with a purely nonlinear power-form restoring force," Journal of Sound and Vibration, vol. 330, no. 17, pp. 4313-4327, 2011.

[12] A. K. Mallik, V. Kher, M. Puri, and H. Hatwal, "On the modelling of non-linear elastomeric vibration isolators," Journal of Sound and Vibration, vol. 219, no. 2, pp. 239-253, 1999.

[13] J. L. Trueba, J. Rams, and M. A. F. Sanjuán, "Analytical estimates of the effect of nonlinear damping in some nonlinear oscillators," International Journal of Bifurcation and Chaos in Applied Sciences and Engineering, vol. 10, no. 9, pp. 2257-2267, 2000.

[14] Alex Elías-Zúñiga and Oscar Martínez-Romero, "Accurate solutions of conservative nonlinear oscillators by the enhanced cubication method," Mathematical Problems in Engineering, vol. 2013, Article ID 842423, 9 pages, 2013.

[15] Alex Elías-Zúñiga and Oscar Martínez-Romero, "Investigation of the equivalent representation form of strongly damped nonlinear oscillators by a nonlinear transformation approach," Journal of Applied Mathematics, vol. 2013, Article ID 245092, 7 pages, 2013. 


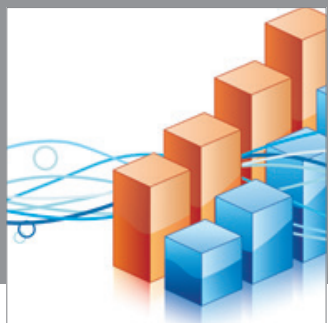

Advances in

Operations Research

mansans

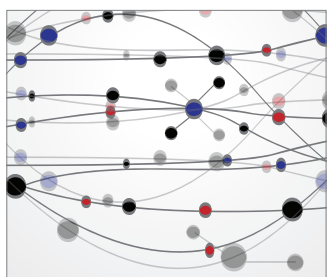

The Scientific World Journal
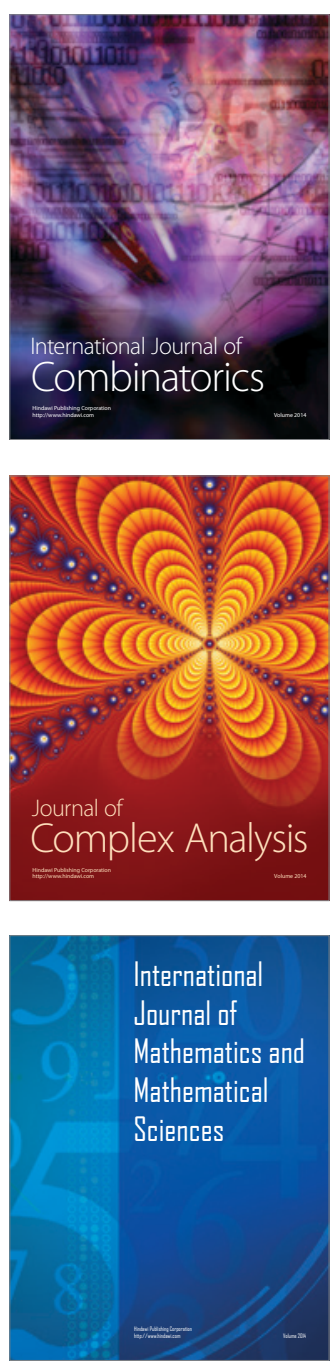
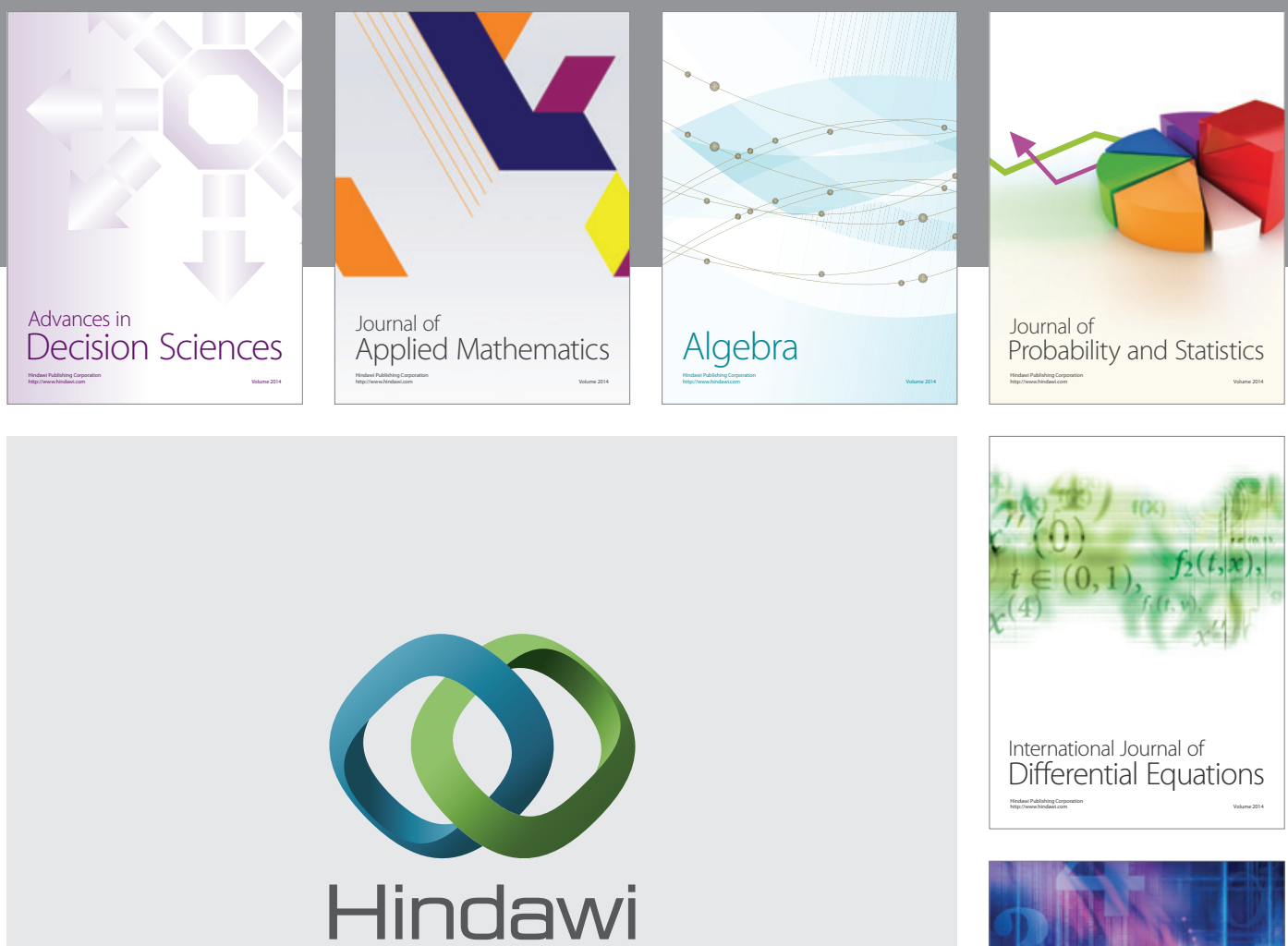

Submit your manuscripts at http://www.hindawi.com
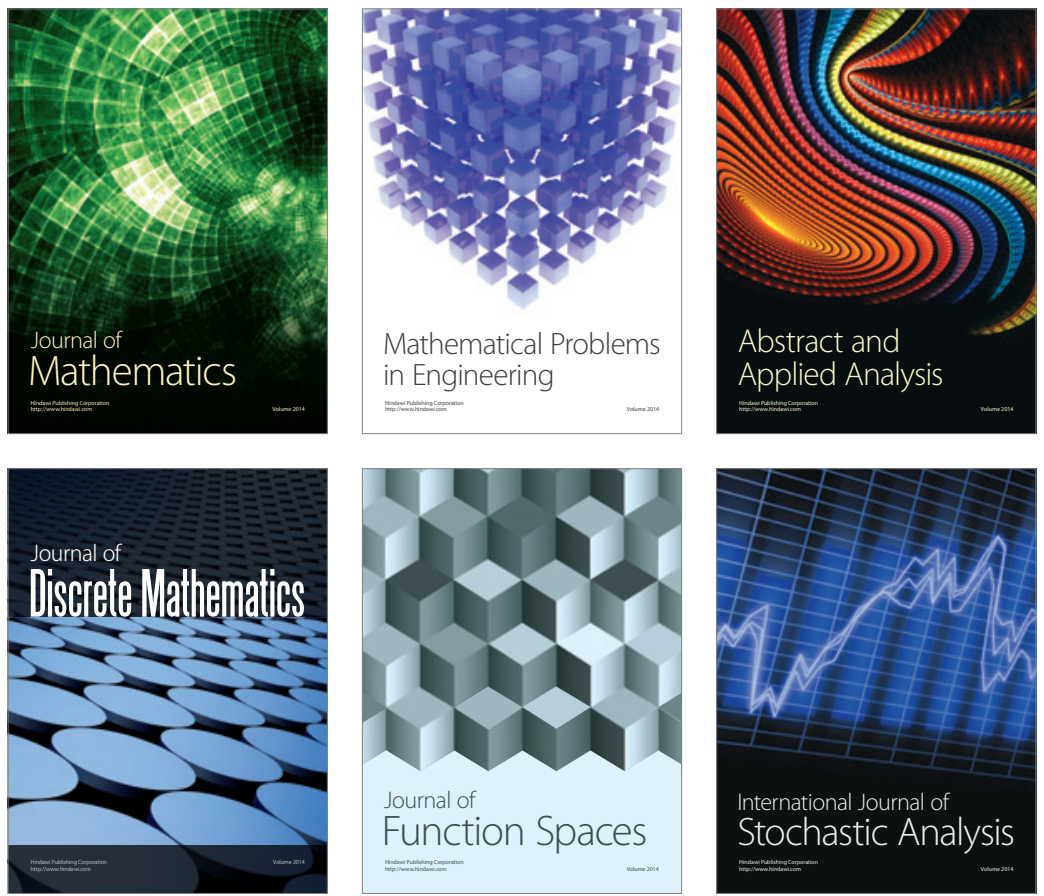

Journal of

Function Spaces

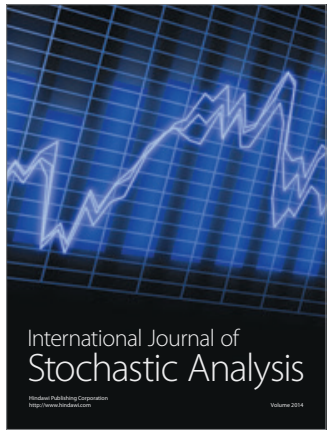

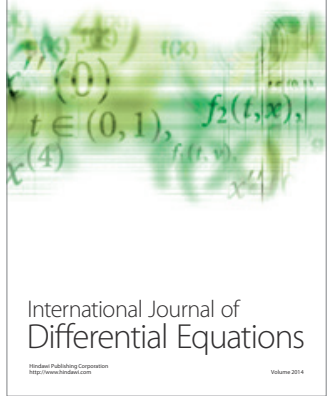
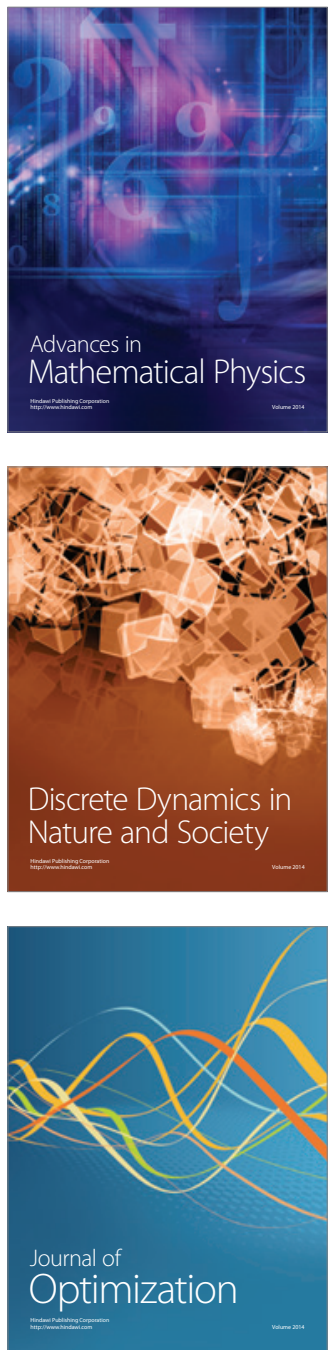